\title{
Statistical cataloging of archival data for luminosity class IV-V stars
}

\section{The epoch 2001 temperature catalog ${ }^{\star}$}

\author{
B. J. Taylor ${ }^{\star \star}$ \\ Department of Physics and Astronomy, N283 ESC, Brigham Young University, Provo, UT 84602-4360, USA
}

Received 2 July 2002 / Accepted 14 November 2002

\begin{abstract}
This paper is one of a pair in which temperatures and metallicity catalogs for class IV-V stars are considered. The temperature catalog described here is derived from a calibration based on stellar angular diameters. If published calibrations of this kind are compared by using color-index transformations, temperature-dependent differences among the calibrations are commonly found. However, such differences are minimized if attention is restricted to calibrations based on Johnson $V-K$. A calibration of this sort from Di Benedetto (1998) is therefore tested and adopted. That calibration is then applied to spectroscopic and photometric data, with the latter predominating. Cousins $R-I$ photometry receives special attention because of its high precision and low metallicity sensitivity. Testing of temperatures derived from the calibration suggests that their accuracy and precision are satisfactory, though further testing will be warranted as new results appear. These temperatures appear in the catalog as values of $\theta \equiv 5040 / T$ (effective). Most of these entries are accompanied by measured or derived values of Cousins $R-I$. Entries are given for 951 stars.
\end{abstract}

Key words. catalogs - stars: fundamental parameters

\section{Introduction}

Some years ago, Taylor (1994c) published an $[\mathrm{Fe} / \mathrm{H}]$ catalog for about 400 class IV-V FGK stars. The input data for the catalog consisted of published values of $[\mathrm{Fe} / \mathrm{H}]$ derived from weak lines, usually by means of high-dispersion analysis. Those data were corrected to a common temperature scale and (as far as possible) to a common zero point. They were then used to calculate mean values of $[\mathrm{Fe} / \mathrm{H}]$ and rms errors.

A second iteration of the catalog appeared in 1995 (see Taylor 1995, hereafter Paper II). For that version, the cutoff date for published values of $[\mathrm{Fe} / \mathrm{H}]$ was the end of 1993 . Of course, numerous high-dispersion metallicities have been published since that time. A third iteration of the catalog is therefore desirable.

The temperature data required for each iteration are published in a catalog of their own. Previous versions of the temperature catalog are discussed in Paper II and by Taylor (1994b, hereafter Paper I). The derivation of an updated temperature catalog is discussed in this paper. An updated $[\mathrm{Fe} / \mathrm{H}]$ catalog is discussed in a companion paper (see Taylor 2003).

* Catalog is only available in electronic form at the CDS via anonymous ftp to cdsarc.u-strasbg.fr (130.79.128.5) or via http://cdsweb.u-strasbg.fr/cgi-bin/qcat?J/A+A/398/721

$\star \star$ e-mail: taylorb@byu.edu
The plan for this paper is as follows. In Sect. 2, temperature calibrations are considered and a choice among them is made. Temperature calculations and the quality of the results are discussed in Sect. 3. The resulting catalog is described briefly in Sect. 4, and a summary of the paper is given in Sect. 5 .

\section{Choosing a temperature calibration}

The temperatures discussed in Papers I and II were derived from a temperature calibration. For this reason, the first task at hand is to find out whether that calibration should be revised. A prudent way to do this is to look for an improved calibration which has a minimal dependence on the properties of model atmospheres. Attention is therefore restricted to calibrations based on angular diameters from the infrared flux method and/or direct measurement.

Temperature-dependent disagreements among calibrations have been common (see, for example, Figs. 1 and 2 of Taylor 1992). A second requirement of a prudent search is therefore a comparison of calibrations. This step is not feasible if the calibrations are simply adopted in their published forms because they are commonly based on diverse color indices. Color-index transformations are therefore applied to restate all the candidate calibrations in terms of Cousins $R-I$, which is a convenient choice for this purpose. The adopted transformations are 


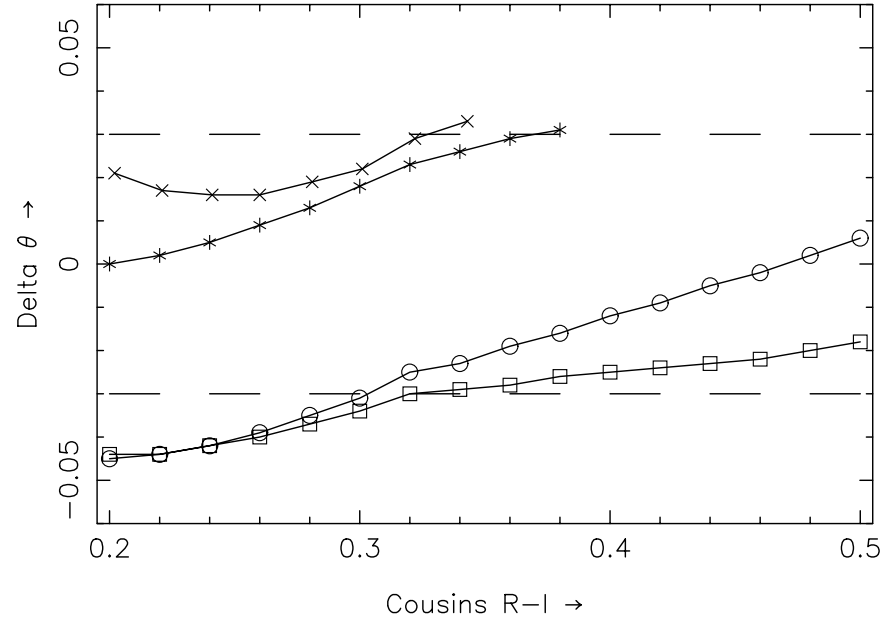

Fig. 1. In the upper panel, difference curves are given for a $\beta$ calibration (crosses) and a Johnson $V-R$ calibration (asterisks). Both calibrations are from Alonso et al. (1996). In the lower panel, difference curves are given for $B-V$ calibrations from Sekiguchi \& Fukugita (2000; open circles) and Alonso et al. (open squares). All four calibrations apply at solar metallicity, and all are differenced from the Di Benedetto (1998) calibration. The values of $\left|\Delta T_{\text {eff }}\right|$ corresponding to $|\Delta \theta|=0.01$ range from $93 \mathrm{~K}$ at $(R-I)_{\mathrm{C}}=0.2$ to $43 \mathrm{~K}$ at $(R-I)_{\mathrm{C}}=0.5$.

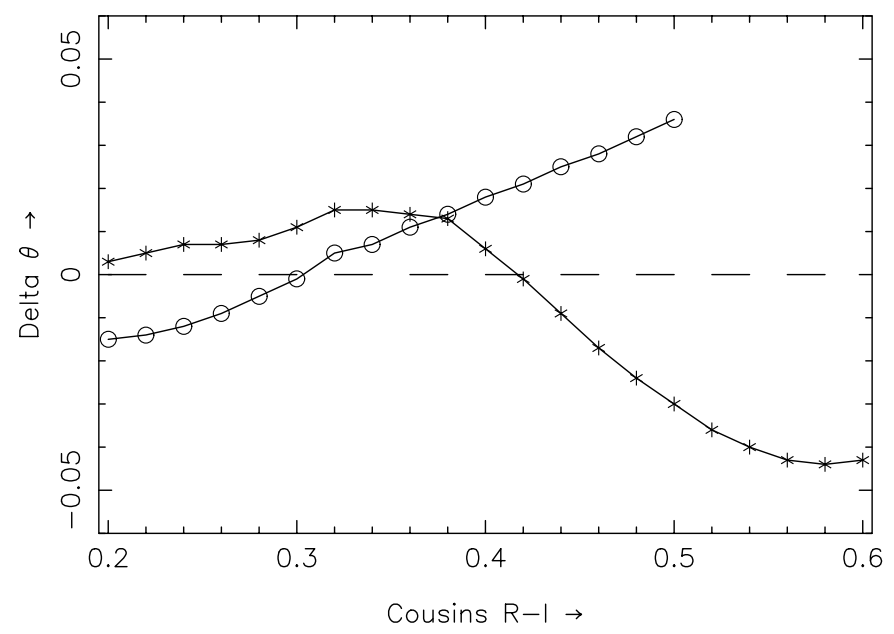

Fig. 2. Difference curves are given for $B-V$ (open circles) and Johnson $R-I$ (asterisks). Both calibrations are from Alonso et al. (1996), apply at solar metallicity, and are differenced from the Di Benedetto (1998) calibration. The values of $\left|\Delta T_{\text {eff }}\right|$ corresponding to $|\Delta \theta|=0.01$ range from $93 \mathrm{~K}$ at $(R-I)_{\mathrm{C}}=0.2$ to $36 \mathrm{~K}$ at $(R-I)_{\mathrm{C}}=0.6$.

given by Taylor (1986) and below in Appendix A, where all transformations used in this paper are discussed.

In Figs. 1-3, difference curves between 11 restated calibrations are plotted as a function of $(R-I)_{\mathrm{C}}$. Details about the plotted curves are given in the figure captions. As one can readily see, the salient features of the curves are their generally nonzero slopes. Both positive and negative slopes appear (see especially Fig. 2). In addition, it is found that the curves can differ substantially. The largest such difference found is about 0.067 in $\theta$ or $286 \mathrm{~K}$ (again see Fig. 2) ${ }^{1}$.

\footnotetext{
${ }^{1}$ In agreement with common practice, $\theta \equiv 5040 / T_{\text {eff }}$.
}

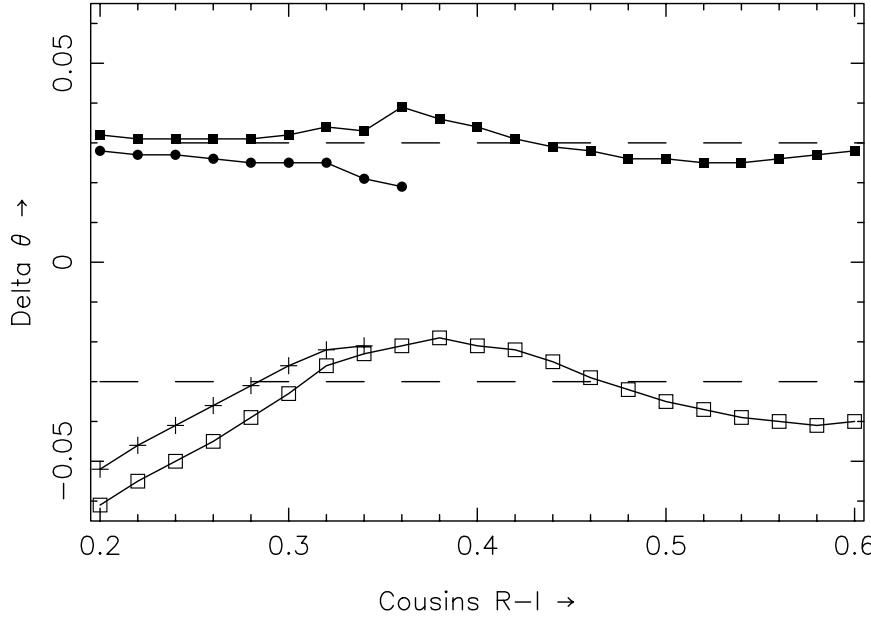

Fig. 3. In the upper panel, difference curves are given for $(V-K)_{\mathrm{J}}$ calibrations. Solid squares apply for a calibration from Alonso et al. (1996), while solid circles apply for a calibration from Fernley (1989). The difference curves have been offset slightly from each other for the sake of clarity. In the lower panel, difference curves are given for a Strömgren calibration (plus signs) and a $(V-I)_{\mathrm{J}}$ calibration (open squares). Both calibrations are from Alonso et al. The first three calibrations apply at solar metallicity, while the $(V-I)_{\mathrm{J}}$ calibration applies if $-0.5 \leq[\mathrm{Fe} / \mathrm{H}] \leq+0.5$. All four calibrations have been differenced from the Di Benedetto (1998) calibration. The values of $\left|\Delta T_{\text {eff }}\right|$ corresponding to $|\Delta \theta|=0.01$ range from $93 \mathrm{~K}$ at $(R-I)_{\mathrm{C}}=0.2$ to $36 \mathrm{~K}$ at $(R-I)_{\mathrm{C}}=0.6$.

Conceivably these disagreements could be attributed to the adopted color-index transformations. However, there is good reason to believe that those transformations are both accurate and precise (again see Appendix A). One might also ask whether the calibrations disagree because they have been derived from diverse sets of temperature data. This possibility may be assessed by considering only a subset of 9 transformations secured from Alonso et al. (1996). Though those authors did in fact use a single set of temperature data, disagreements appear among their calibrations. In particular, the differences between the curves plotted in Fig. 2 are based exclusively on the Alonso et al. results.

It would ultimately be interesting to find out exactly how these disagreements arise. Meanwhile, it is possible to avoid the problem altogether. One begins by setting aside all calibrations based on the obsolete Johnson VRI system. When the remaining calibrations are examined, those based on Johnson $V-K$ attract notice at once, since they seem to be less affected by overall slope differences than the others (see the upper panel of Fig. 3). One of those calibrations has been given by Fernley (1989) and does not apply for late-type stars (see the lower of the two lines in the upper panel of Fig. 3). The remaining options are calibrations given by Alonso et al. and by Di Benedetto (1998). The latter is adopted for further consideration, though with the admission that there seems to be little to choose between the two calibrations.

In Fig. 4, the Di Benedetto calibration is compared to the Taylor (1992) calibration, which was used in Papers I and II. The latter is expressed in terms of $(R-I)_{\mathrm{C}}$, so a transformation between that index and $(V-K)_{\mathrm{J}}$ is employed. The circled 


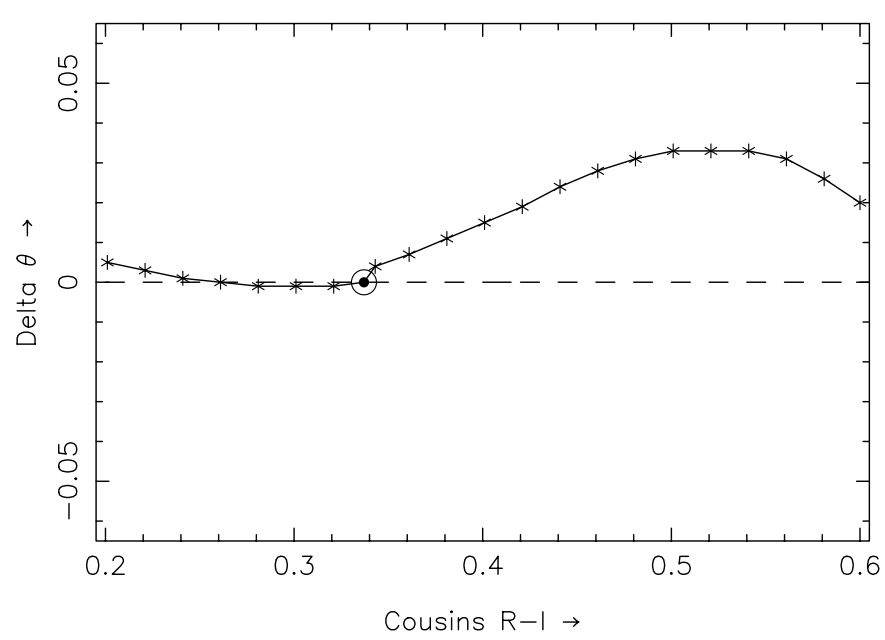

Fig. 4. The plotted curve represents the difference between the Di Benedetto (1998) and Taylor (1992) calibrations. The circled dot represents the position of the Sun (see Appendix B for pertinent data). The values of $\left|\Delta T_{\text {eff }}\right|$ corresponding to $|\Delta \theta|=0.01$ range from $93 \mathrm{~K}$ at $(R-I)_{\mathrm{C}}=0.2$ to $36 \mathrm{~K}$ at $(R-I)_{\mathrm{C}}=0.6$.

Table 1. Adopted temperature calibration ${ }^{\mathrm{a}}$.

\begin{tabular}{ccl}
\hline \hline $\begin{array}{c}\text { Lower } \\
(R-I)_{\mathrm{C}}\end{array}$ & $\begin{array}{c}\text { Upper } \\
(R-I)_{\mathrm{C}}\end{array}$ & Relation \\
\hline 0.201 & 0.260 & $\theta=0.975(R-I)_{\mathrm{C}}+0.538$ \\
0.260 & 0.335 & $\theta=1.075(R-I)_{\mathrm{C}}+0.512^{\mathrm{b}}$ \\
0.335 & 0.501 & $\theta=1.281(R-I)_{\mathrm{C}}+0.443$ \\
0.501 & 0.621 & $\theta=-0.123+3.633(R-I)_{\mathrm{C}}-2.44(R-I)_{\mathrm{C}}^{2}$ \\
\hline
\end{tabular}

${ }^{a}(R-I)_{\mathrm{C}}$ is given below in magnitudes, while $\theta$ is unitless.

b This part of the calibration is adapted from a relation given by Taylor (1992). That relation agrees with the Di Benedetto calibration in the stated color range.

dot in Fig. 4 represents the position of the Sun. For stars hotter than the Sun, acceptable agreement between the two calibrations is found. For cooler stars, however, one can see that the calibrations diverge.

The cause of this disagreement appears to reside in the data bases used to derive the two calibrations. Taylor (1992; see his Fig. 3) had few data available for stars with $(R-I)_{\mathrm{C}}>0.4$. However, substantial numbers of such data are now available (see, for example, the part of Fig. 4 in Di Benedetto 1998 for which $\left.(V-K)_{\mathrm{J}}>1.84\right)$. It therefore seems advisable to take advantage of this improved data base by adopting the Di Benedetto calibration.

The adopted temperature relation is given in Table 1, with $(R-I)_{\mathrm{C}}$ being used as the argument. For the temperature range given in the table, the maximum difference between the Table 1 polynomials and the Di Benedetto calibration is $13 \mathrm{~K}$. For solar-type stars, this corresponds to $|\Delta \theta|=0.002$, which is smaller than the rms errors of almost all values of $\theta$ in the catalog.

For $\theta>0.935$, the Table 1 relation may be checked by using Balmer-line temperatures given by England (1980), Cayrel de Strobel \& Cayrel (1989), and Fuhrmann (1998).
Those data are adopted because they have all been zeroed to the Sun. An $[\mathrm{Fe} / \mathrm{H}]$ correction is applied to them (see Eq. (A.16), Table A.4, Appendix A). Once this is done, they yield the following formal correction to the Table 1 relation:

$10^{3} \Delta \theta=-0.8 \pm 3.8$,

corresponding to $-4 \pm 19 \mathrm{~K}$ at $5000 \mathrm{~K}$.

It should be noted that Eq. (1) is not definitive. A decisive assessment of temperatures for $\mathrm{K}$ dwarfs will be possible only when angular diameters have been measured for an appreciable number of such stars. Meanwhile, it seems fair to say that for cool as well as hot stars, tests of the Table 1 relation yield encouraging results. Accordingly, that relation is adopted.

\section{Deriving temperatures}

\subsection{The role of Cousins $R-I$}

As in Papers I and II, most temperatures are determined from data on a particular photometric system. The system of choice continues to be $(R-I)_{\mathrm{C}}$. To allow for the small (but apparently nonzero) blanketing sensitivity of that color index, the model-atmosphere results of Buser \& Kurucz (1992) are used ${ }^{2}$. This procedure differs from that in Papers I and II, where no allowance for the blanketing sensitivity of $(R-I)_{\mathrm{C}}$ was made.

Besides its small blanketing sensitivity, $(R-I)_{\mathrm{C}}$ commonly offers the advantage of high precision. This feature may be illustrated by comparing temperature errors from $(R-I)_{\mathrm{C}}$ and $(V-K)_{\mathrm{J}}$. If the rms error for $(V-K)_{\mathrm{J}}$ is $15 \mathrm{mmag}$ (see Campins et al. 1985), the Di Benedetto calibration yields a corresponding rms temperature error of $20 \mathrm{~K}$ for a solar-type star. For $(R-I)_{\mathrm{C}}$, the best available rms error is $3 \mathrm{mmag}$ (see Appendix B of Taylor 1996). The corresponding temperature error is $21 \mathrm{~K}$. This comparison is meant to be illustrative, since results in both systems have a range of rms errors. However, it does seem fair to say that the errors from the two systems can be comparable ${ }^{3}$.

\subsection{The role of other photometric and spectroscopic data}

For many of the stars listed in the catalog, $(R-I)_{\mathrm{C}}$ has not been measured. As in Papers I and II, most of the temperature data for such stars are derived from photometry in other systems. Preference is given to results in systems with high precision and low blanketing sensitivity. The transformations used to derive values of $(R-I)_{\mathrm{C}}$ from such data are given in Appendix A (see below). Many of the adopted data sources are listed in Tables 1 and 4 of Paper I. The remainder are cited in the Lausanne photometric data base (Mermilliod et al. 1997) or in the footnotes to the tables in Appendix A.

\footnotetext{
2 The resulting correction equation is Eq. (A.16) in Table A.4 of Appendix A.

3 A 3-mmag rms error can be obtained by making four measurements of $(R-I)_{\mathrm{C}}$ with a single photometer (see Cousins 1980b). By contrast, measurements of $V-K$ require different photometers for $V$ and $K$. For investigations like those of Alonso et al. and Di Benedetto, measurements of $(R-I)_{\mathrm{C}}$ instead of $V-K$ should therefore be considered.
} 
Table 2. Formal corrections to published temperatures: first set.

\begin{tabular}{|c|c|c|c|}
\hline Source & $\begin{array}{l}\text { Algebraic } \\
\text { difference }^{\mathrm{a}}\end{array}$ & $\begin{array}{l}\text { Numerical } \\
\text { difference }^{\text {b }}\end{array}$ & $\begin{array}{c}10^{3} \sigma(\theta) \\
(\text { derived) }\end{array}$ \\
\hline Fulbright (2000) & $10^{3}\left(\theta-\theta_{\mathrm{F}}\right)$ & $2.6 \pm 3.3$ & $15.2 \pm 2.3$ \\
\hline Gonzalez et al. ${ }^{\mathrm{d}}$ & $10^{3}\left(\theta-\theta_{\mathrm{G}}\right)$ & $1.8 \pm 2.0$ & $9.3 \pm 1.5$ \\
\hline Kobi \& North (1990) & $10^{3}\left(\theta-\theta_{\mathrm{K}}\right)$ & $-1.1 \pm 1.5$ & $5.8 \pm 1.6$ \\
\hline Santos et al. $(2001)^{\mathrm{e}}$ & $10^{3}\left(\theta-\theta_{\mathrm{S}}\right)$ & $8.5 \pm 2.0$ & $9.1 \pm 1.6$ \\
\hline Thoren \& Feltzing (2000) & $10^{3}\left(\theta-\theta_{\mathrm{F}}\right)$ & $5.4 \pm 2.6$ & $5.1 \pm 1.5$ \\
\hline
\end{tabular}

a " $\theta$ " designates photometric results from this paper.

b The entries in this column are numerical values of the algebraic expressions just to the left. The quoted error bars are rms errors.

c These entries have been derived by using the comparison algorithm described in the text. They apply to the values of $\theta$ from the sources cited in the first column.

d Contributing papers are Gonzalez (1998), Gonzalez \& Vanture (1998), Gonzalez et al. (1999), Gonzalez \& Laws (2000), and Gonzalez et al. (2001).

e The entries to the right apply if $\theta_{\mathrm{S}} \leq 0.885$. If $\theta_{\mathrm{S}} \geq 0.9, \theta=$ $(1.336 \pm 0.044) \theta_{\mathrm{S}}-(0.306 \pm 0.042)$.

For some stars, neither values of $(R-I)_{\mathrm{C}}$ nor data on other preferred photometric systems can be found. $B-V$ is not used in these cases except for one star with near-Hyades abundances. In that case, the required correction for differential blanketing is acceptably small. That correction is derived from the BuserKurucz results.

For stars without other temperature data, temperatures from the Kobi-North (1990) calibration of Geneva photometry are adopted when possible. Otherwise, temperatures derived from high-dispersion analyses of weak lines are used. For both kinds of data, rms errors must be derived and tests must be made for possible systematic corrections. These results are obtained by comparing the published data to photometric counterparts on the Table 1 system. If a correction equation with a scale factor differing from unity is required, a two-error least-squares analysis is performed (Madansky 1959). If only a zero-point offset is required, it is obtained from a "comparison algorithm" which is also used to calculate rms errors. The comparison algorithm is given in Appendix A of Taylor (1991), and is described conceptually in Sect. 4.3 of Taylor (1999).

Results obtained from these analyses are summarized in Table 2. The correction equations for the weak-line temperatures are diverse enough to suggest that such data should not be used to check the adopted calibration (see especially note "e" of Table 2). However, one also finds that weak-line temperatures can have rms errors of about $70 \mathrm{~K}$ or less (see the second, fourth, and fifth entries in the fourth column of the table). They are therefore adequate for present use.

\subsection{Reddening and binaries}

Discussions of these issues have been given in Papers I and II and require only a brief summary here. For binaries, a procedure which is described in Sect. 4 of Paper I is applied. No reddening corrections to the derived temperatures are made because reddening near the Sun is known to be very small from polarimetric results (see point 4 of Sect. 3 in Paper II).

\subsection{The accuracy and precision of the derived temperatures}

The rms errors of the derived temperatures are worth close attention because they contribute to the rms errors quoted in the $[\mathrm{Fe} / \mathrm{H}]$ catalog. As in Papers I and II, the temperature errors are obtained largely from the rms errors of the contributing photometry. An allowance for the rms zero-point error of the adopted calibration is included (see Sects. 3.1 and 6 of Paper I and Appendix B of this paper).

The quality of the catalog temperatures was discussed in some detail in Paper II. An updated discussion is now required, however, by the appearance of a pertinent analysis performed by Fuhrmann et al. (1994, hereafter F94). Those authors derive temperatures from Balmer-line profiles and then compare their results to photometric temperatures. They find that the latter have a large scatter and can suffer from serious systematic errors. F94 go so far as to suggest that those problems are due in part to faulty reduction procedures applied to broad-band photometry.

F94 did not test the Paper I temperature data. The feasibility of that kind of test has since been increased because Fuhrmann (1998, hereafter F98) has published an additional set of Balmer-line temperatures. To perform the test, metallicity corrections will be applied to both the F94 and F98 data (again see Eq. (A.16), Table A.4, Appendix A of this paper). The comparison algorithm mentioned in Sect. 3.2 will also be used.

The results of the tests are given in the entries for F94 and F98 in Table 3. Details about the entries in Table 3 are given in the table's footnotes. The fourth and fifth columns of the table are of particular interest here. If the excessive scatter found by F94 were present in the catalog temperatures, the comparison algorithm would yield spuriously large rms errors for the F94 and F98 temperatures (see Col. 4). Those entries would be substantially larger than the errors quoted by F94 and F98 (see the non-boldface entries in Col. 5). In fact, the errors from the comparison algorithm appear to be smaller than the errors quoted by F94 and F98.

For each program star they consider, F94 present results from as many as four Balmer lines. Data from two or more lines are available for a number of their program stars. From the scatter in those data, an rms error may be derived for the mean F94 temperatures. That rms error is given in boldface in Col. 5 of Table 3. Comparisons with the non-boldface entries just below and just above it suggest that F94 (and presumably F98 as well) have overestimated the rms errors which they themselves quote. However, if the boldface entry is compared to the Col. 4 entries from the comparison algorithm, no differences as large as $2 \sigma$ are found. As a result, the comparisons do not suggest that the catalog rms errors are too large. 
Table 3. Formal corrections to published temperatures: second set.

\begin{tabular}{lcccc}
\hline \hline Source & Algebraic difference $^{\mathrm{a}}$ & $\begin{array}{c}\text { Numerical } \\
\text { difference }^{\mathrm{b}}\end{array}$ & $\begin{array}{c}10^{3} \sigma(\theta) \\
(\text { derived })^{\mathrm{c}}\end{array}$ & $\begin{array}{c}10^{3} \sigma(\theta) \\
(\text { source })^{\mathrm{d}}\end{array}$ \\
\hline $\begin{array}{l}\text { F98 } \\
\text { (Balmer lines) }\end{array}$ & $10^{3}\left(\theta-\theta_{\mathrm{F}}\right)+25[\mathrm{Fe} / \mathrm{H}]^{\mathrm{e}}$ & $-1.8 \pm 1.4$ & $6.9 \pm 1.2$ & 11 \\
$\begin{array}{l}\text { F94 } \\
\text { (Balmer lines) }\end{array}$ & $10^{3}\left(\theta-\theta_{\mathrm{F}}\right)+25[\mathrm{Fe} / \mathrm{H}]^{\mathrm{f}}$ & $-33 \pm 2$ & $8.0 \pm 2.3$ & $\begin{array}{c}\mathbf{4 . 6}^{\mathrm{g}} \\
15\end{array}$ \\
$\begin{array}{l}\text { Gray (1994) } \\
\text { (line-depth ratios) }\end{array}$ & $10^{3}\left(\theta-\theta_{\mathrm{G}}^{\prime}\right)+31[\mathrm{Fe} / \mathrm{H}]^{\mathrm{h}}$ & $\ldots$. & $8.6 \pm 1.1^{\mathrm{j}}$ & $2.1 \pm 1.6^{\mathrm{j}}$ \\
\hline
\end{tabular}

a Unsubscripted values of $\theta$ designate catalog results. Subscripted values are from the sources cited in the first column.

b The entries in this column are numerical values of the algebraic expressions just to the left. The quoted error bars are rms errors of means.

c The entries in this column apply to unsubscripted values of $\theta$ (see note "a"). The quoted errors are rms errors of $10^{3} \sigma(\theta)$.

d Entries without footnotes are published rms errors.

e The range of tested values of $\theta$ is $\leq 0.891$. For the numerical coefficient of $[\mathrm{Fe} / \mathrm{H}], \sigma=8 \mathrm{dex}^{-1}$.

${ }^{\mathrm{f}} \theta_{\mathrm{F}}$ is on a zero point defined by $\mathrm{H} \beta, \mathrm{H} \gamma$, and $\mathrm{H} \delta$ measurements. $\mathrm{H} \alpha$ measurements are included with a zero-point adjustment of $+46 \pm 7 \mathrm{~K}$. For the numerical coefficient of $[\mathrm{Fe} / \mathrm{H}], \sigma=8 \mathrm{dex}^{-1}$.

$\mathrm{g}$ This number has been derived from the scatter in results presented for four Balmer lines by F94. A variance has been obtained from that scatter for each star which contributes to the calculation. The set of variances has been averaged, and the quoted rms error is the square root of that variance.

h $\theta_{\mathrm{G}}^{\prime}=(0.92 \pm 0.03) \theta_{\mathrm{G}}+(0.067 \pm 0.002)$, with $\theta_{\mathrm{G}} \equiv 5040 / T$ (Gray). For the numerical coefficient of $[\mathrm{Fe} / \mathrm{H}], \sigma=9 \mathrm{dex}^{-1}$.

j This result is derived from multiple data entries. The procedure parallels that described in footnote "e". An $F$ test shows that at $98 \%$ confidence, the rms error from Gray's errors differs from the rms error deduced from the comparison algorithm.

An analysis of accuracy also yields a conclusion which contrasts with that of F94. Before that conclusion is stated, it is worth noting that if only temperatures from Balmer-line profiles are considered and that if those temperatures have been zeroed to the Sun, they agree reasonably well with those from the adopted calibration. This result was established by Taylor (1992, 1997), and is supported by Eq. (1) (recall Sect. 2). To see whether the agreement extends to the F98 results, a mean difference between those results and the catalog data may be consulted. That difference is given in the first entry in the third column of Table 3, and one can see at once that it does not differ from zero at $95 \%$ confidence 4 .

Tests of the F94 data have previously been given in Sect. 7 and Table 1 of Taylor (1997). Taylor found that those data are on two different zero points which differ at better than $6 \sigma$ confidence (see footnote "f" of Table 3). In addition, he found that if no metallicity corrections are applied to the F94 data, they yield an anomalous value of $(R-I)_{\mathrm{C}}$ for the Sun. If a metallicity correction is applied to the F94 temperatures, one now finds that they are still anomalous. Using a zero point for lines other than $\mathrm{H} \alpha$, the F94 data are found to differ from the catalog data by about $-245 \mathrm{~K}$. This difference is significant at a $16 \sigma$ confidence level. If an $\mathrm{H} \alpha$ zero point is used instead, the offset becomes $-291 \mathrm{~K}$ and is significant at a $20 \sigma$ confidence level.

F94, unlike F98, give no indication that their data have been zeroed to the Sun. With this in mind, the results of the

4 It should be noted that for the temperature range of interest, the revised calibration differs modestly (if at all) from its predecessor. As a result, conclusions cited above that were drawn by using the predecessor turn out to be unchanged when the revised calibration is adopted instead.
Table 4. A sample of the temperature catalog ${ }^{\mathrm{a}}$.

\begin{tabular}{lrcrccccc}
\hline \hline Prefix & Number & Prefix & Number & $(R-I)_{\mathrm{C}}$ & $\sigma$ & $\theta$ & $\sigma$ \\
\hline HD & 400 & HR & 17 & 0.293 & 0.007 & 0.826 & 0.008 \\
HD & 693 & HR & 33 & 0.290 & 0.003 & 0.823 & 0.003 \\
HD & 739 & HR & 35 & 0.252 & 0.007 & 0.783 & 0.007 \\
HD & 1237 & Hic & 1292 & 0.373 & 0.003 & 0.919 & 0.003 \\
HD & 1461 & HR & 72 & 0.340 & 0.003 & 0.878 & 0.004 \\
\hline
\end{tabular}

a $(R-I)_{\mathrm{C}}$ is given below in magnitudes, while $\theta$ is unitless. In the full catalog, suffixes "A" and "B" are added to the first catalog number to designate components of binaries (when necessary).

numerical tests suggest that $a$ ) the F94 (but not the F98) data suffer from systematic offsets, $b$ ) the differing offsets between the F94 $\mathrm{H} \alpha$ data and their data for other Balmer lines are one manifestation of this problem, and c) the F94 results therefore do not present a valid reason for questioning the zero point of the catalog data ${ }^{5}$.

Proceeding to the third line of Table 3 , one encounters results for a second pertinent data set. That data set has been published by Gray (1994) and was discussed in Sect. 3 of Paper II. A revised analysis of the Gray data yields an apparent scalefactor difference between those data and results from the updated calibration (see footnote "h" of Table 3). An analysis of rms errors, however, yields a conclusion which is similar to that of Paper II: when the Gray data are compared to catalog data,

\footnotetext{
5 A referee has suggested that the problem with the F94 temperatures is related to the value of the mixing length which F94 apply in their model atmospheres.
} 
the resulting scatter is too large to be explained by the combined effect of rms errors from the two sources (see the third entry in Table 3 and footnotes " $h$ " and "j" of the table).

The overall conclusion drawn here is that the addition of the F98 results to the discussion has made it appear more likely that the catalog rms errors are correct. The catalog errors will therefore be adopted, but with an acknowledgment that further testing will be necessary in the future.

\section{A brief description of the catalog}

The catalog is available in electronic form from the Strasbourg Astronomical Data Center (CDS). There are entries in the catalog for 951 stars. A sample of the catalog is given in Table 4.

It is worthwhile to make two comments about the catalog for the benefit of potential users. Compilers of photometric catalogs should note that the catalog values of $(R-I)_{\mathrm{C}}$ are either quoted from primary sources or derived from other photometric systems. For this reason, they should not be included in compilations of photometry from primary sources. Analysts of the $[\mathrm{Fe} / \mathrm{H}]$ data base are invited to select temperature-defined samples by using the catalog entries. That procedure should be superior to using $B-V$, as Haywood (2001) has done, because the catalog values of $\theta$ are effectively insensitive to metallicity.

\section{Summary}

The construction of a temperature catalog for 951 class IV-V stars has been described. The catalog is based on temperature calibrations using $(R-I)_{\mathrm{C}}$ and $(V-K)_{\mathrm{J}}$, since those arguments appear to yield the most satisfactory results. The adopted calibration is a combination of calibrations by Taylor (1992) and Di Benedetto (1998). To derive temperatures, this calibration is applied to values of $(R-I)_{\mathrm{C}}$ from direct measurement and from other photometric systems. The accuracy and precision of the resulting temperatures are deemed to be satisfactory, though further testing will be advisable as new results appear.

Acknowledgements. Phil Warner set up for my use the plot package used to produce the figures in this paper. Mike and Lisa Joner proofread the paper carefully, and two anonymous referees made a number of constructive suggestions for improving the paper. I cheerfully thank all these individuals while noting that page charges for this paper have been generously underwritten by the College of Physical and Mathematical Sciences and the Physics and Astronomy Department of Brigham Young University.

\section{Appendix A: Color-index transformations}

The color-index transformations that are applied in this paper may be divided into two groups. One of these groups includes transformations between "quasi-Johnson" $V-R$ and $R-I$ and their Cousins counterparts. These transformations are based on data from Johnson et al. (1966) and Cousins (1980a, 1980b). The transformations are given in Table 4 of Taylor (1986), and require no revision.

The transformations in a second group are scattered through a number of papers other than Taylor (1986). Some members of this group do require revision. To take care of that problem while listing the members of the group in a single place for the sake of convenience, they are stated (in updated form, when necessary) in the following tables.

- Table A.1 lists transformations from $\beta, \alpha$, and $b-y$ to $(R-$ $I)_{\mathrm{C}}$.

- Tables A.2 and A.3 list zero points for the relations in Table A.1 and also rms errors for values of $(R-I)_{\mathrm{C}}$ that are derived from the Table A.1 relations.

- Table A.4 lists transformations and other equations of interest that do not include $\beta, \alpha$, or $b-y$.

Many of the data used to derive these transformations are from sources listed in the Lausanne data base (Mermilliod et al. 1997). The remaining data are from sources listed in the footnotes to Tables A. 1 and A.4.

Color-index transformations have been controversial in the past, and they and other least-squares relations are not always documented properly in the literature. Three criteria are therefore given below to help readers form quick estimates of the reliability of transformations. Comments about the transformations presented here and in Taylor (1986) are also given.

1. If transformations between color indices are to be feasible, they must not be influenced unduly by strong absorption features. Most such problems appear for M stars (see Taylor \& Joner 1996 for an example). However, M stars are not considered in this context. For earlier-type stars, the Paschen jump turns out to be the only absorption feature of concern. The adopted transformation between the Cousins and quasi-Johnson $R-I$ systems does include a Paschen-jump term (see the third transformation for the "quasi-Johnson" system in Table 4 of Taylor 1986). No such term is required for the other transformations of interest here ${ }^{6}$.

2. If the rms error of the centroid of a transformation is about 0.003 or less (for $\theta$ ) or 3 mmag or less (for color indices), it is likely that enough data have been used to define the transformation precisely. For the transformations considered here, the only exception to this rule is Eq. (A.13) (see Table A.4). The rms error for the centroid of that transformation is $5.4 \mathrm{mmag}$, and reflects the relatively large rms errors of the contributing values of $(V-K)_{\mathrm{J}}$. Fortunately, that error is still markedly smaller than rms errors for values of $(V-K)_{\mathrm{J}}$ inferred from the transformation (for an example, see Sect. 7 of Taylor 1997).

3. Let $Q$ be a coefficient in a transformation, $\sigma_{\mathrm{Q}}$ be its rms error, and $t=|Q| \sigma_{\mathrm{Q}}^{-1}$. A quick way to check the statistical significance of a coefficient is to see whether its value of $t \geq 2$, while noting that coefficients with $t<2$ may sometimes be adopted for the sake of caution. For all terms in the transformations given here, $t>2$.7. The adopted Taylor (1986) transformations include one zero point with $t=1.7$. For other terms in those transformations, $t>10$.

6 Presumably without intending to do so, Alonso et al. have supplied a convenient illustration that one must be careful about Paschenjump effects. Those authors have evaluated the Taylor (1992) calibration by using a transformation from Bessell (1983) which does not allow for the Paschen-jump effect. 
Table A.1. Transformations to $(R-I)_{\mathrm{C}}$ from $\beta, \alpha$, and $b-y^{\mathrm{a}}$.

\begin{tabular}{|c|c|c|}
\hline $\begin{array}{l}\text { Equation } \\
\text { number }\end{array}$ & Limits $^{\mathrm{b}}$ & Relation \\
\hline A. $1^{\mathrm{c}}$ & $0.00,0.13$ & $\begin{aligned}(R-I)_{\mathrm{C}} & =0.0142[\mathrm{Fe} / \mathrm{H}]+0.194+0.868(\delta \beta)+2.11(\delta \beta)^{2}+Z(\beta) \\
& \pm 0.0047\end{aligned}$ \\
\hline A. $2^{\mathrm{d}}$ & $0.30,0.56$ & $\begin{aligned}(R-I)_{\mathrm{C}} & =0.622-0.901 \alpha+Z(\alpha) \\
& \pm 0.009 \quad 0.021\end{aligned}$ \\
\hline A. $3^{\mathrm{e}}$ & $0.11,0.52$ & $\begin{aligned}(R-I)_{\mathrm{C}} & =0.027+0.772(b-y)_{\mathrm{H}}+A[\mathrm{Fe} / \mathrm{H}]+Z(b-y) \\
& \pm 0.0030 .009\end{aligned}$ \\
\hline
\end{tabular}

a Units are dex for $[\mathrm{Fe} / \mathrm{H}]$ and magnitudes for all other quoted variables. An rms error is stated below each calculated numerical coefficient. No errors are stated below adopted coefficients. The original versions of these transformations are given in Tables 2 and 3 of Paper I.

b The stated numbers are lower and upper limits for $\beta, \alpha$, or $b-y$.

c $\delta \beta \equiv 2.720-\beta$. Coefficients without rms errors have been adapted from Crawford (1975). For field-star values of $Z$, see Eq. (A.4) in Table A.2. For Hyades and Coma values of $Z$, see Eq. (A.8) in Table A.3.

d Values of $\alpha$ are from Raff (1976), Spinrad \& Taylor (1969), and Taylor (1970, 1978, 1980). For field-star values of Z, see Eqs. (A.5) and (A.6) in Table A.2. For Hyades and Coma values of $Z$, see Eq. (A.9) in Table A.3.

e $(b-y)_{\mathrm{H}} \equiv(b-y) \times(1-0.119[\mathrm{Fe} / \mathrm{H}])$. See Eq. (A.15), Table A.4, for an equation for A. For field-star values of $Z$, see Eq. (A.7) in Table A.2. For Hyades and Coma values of $Z$, see Eq. (A.10) in Table A.3.

Table A.2. Field stars: zero points and rms errors for equations in Table A.1.

\begin{tabular}{|c|c|c|c|}
\hline $\begin{array}{c}\text { Equation } \\
\text { number }\end{array}$ & Quantity & $\begin{array}{c}\text { RA range } \\
\text { (hours) }\end{array}$ & Entry $^{\mathrm{a}}$ \\
\hline A. 4 & $\begin{array}{c}Z(\beta) \\
\sigma(R-I)_{\mathrm{C}}^{\mathrm{b}}\end{array}$ & $\begin{array}{c}0-24 \\
-\end{array}$ & $\begin{array}{c}-\mathbf{2 . 0} \pm 1.1 \\
10.9\end{array}$ \\
\hline A. $5^{\mathrm{c}}$ & $\begin{array}{c}Z(\alpha) \\
Z(\alpha) \\
\sigma(R-I)_{\mathrm{C}}^{\mathrm{b}}\end{array}$ & $\begin{array}{c}0-15.9 \\
16.0-24 \\
-\end{array}$ & $\begin{array}{c}-2.0 \pm 1.4 \\
\mathbf{5 . 5} \pm 1.5 \\
4.0\end{array}$ \\
\hline A. $6^{\mathrm{d}}$ & $\begin{array}{c}Z(\alpha) \\
Z(\alpha) \\
\sigma(R-I)_{\mathrm{C}}^{\mathrm{b}}\end{array}$ & $\begin{array}{c}0-14.8 \\
14.9-24 \\
-\end{array}$ & $\begin{array}{c}Z(\mathrm{RA})^{\mathrm{e}} \\
0.7 \pm 1.4 \\
8.0\end{array}$ \\
\hline A. 7 & $\begin{array}{c}Z(b-y) \\
\sigma(R-I)_{\mathrm{C}}^{\mathrm{b}}\end{array}$ & $\begin{array}{c}0-24 \\
-\end{array}$ & $\begin{array}{c}-0.8 \pm 2.0 \\
9.8\end{array}$ \\
\hline
\end{tabular}

a All values of $Z$ and $\sigma$ are in mmag, with $1 \mathrm{mmag}=0.001 \mathrm{mag}$. Only values of $Z$ given in boldface are treated as non-zero.

b These entries are rms errors for values of $(R-I)_{\mathrm{C}}$ determined from $\beta, \alpha$, or $b-y$. An elementary form of the comparison algorithm has been used to determine these errors.

c This group of entries applies for data from Spinrad \& Taylor (1969) and Taylor (1970).

d This group of entries applies for data from Raff (1976) and Taylor (1978, 1980).

e $Z($ RA $)=-(0.71 \pm 0.29) \times($ right ascension in decimal hours $)$.

It should be noted that $t>3$ for two $[\mathrm{Fe} / \mathrm{H}]$ terms that appear for the first time in transformations used for the temperature catalog. Another test of the first of those terms (see Eq. (A.1) in Table A.1) may be made by combining it with the value of $A$ in Eq. (A.15) (see Table A.4). The result is a value of $\partial \beta / \partial[\mathrm{Fe} / \mathrm{H}]$, and is found to be $15.4 \pm 3.6 \mathrm{mmag} \mathrm{dex}^{-1}$ at $T$ (eff) $=6000 \mathrm{~K}$. This number agrees within $2 \sigma$ with the corresponding Alonso et al. value of $20 \mathrm{mmag}_{\mathrm{dex}}{ }^{-1}$.
Table A.3. Hyades and Coma: zero points and rms errors for equations in Table A.1.

\begin{tabular}{cccc}
\hline \hline $\begin{array}{c}\text { Equation } \\
\text { number }\end{array}$ & Quantity & Hyades $^{\mathrm{a}}$ & Coma $^{\mathrm{a}}$ \\
\hline A.8 & $Z(\beta)$ & $2.5 \pm 1.7$ & $-3.4 \pm 3.1$ \\
& $\sigma(R-I)_{\mathrm{C}} \mathrm{b}$ & 6.5 & 9.4 \\
A.9 & $Z(\alpha)$ & $1.2 \pm 1.4$ & $-\mathbf{1 0 . 1} \pm 2.6$ \\
& $\sigma(R-I)_{\mathrm{C}} \mathrm{b}$ & 5.4 & 7.9 \\
A.10 & $Z(b-y)_{\mathrm{C}} \mathrm{b}$ & $-1.9 \pm 1.3$ & $-1.2 \pm 2.1$ \\
& $\sigma(R-I)^{\mathrm{b}}$ & 4.8 & 6.4 \\
\hline
\end{tabular}

a All values of $Z$ and $\sigma$ are in mmag, with $1 \mathrm{mmag}=0.001 \mathrm{mag}$. Only values of $Z$ given in boldface are treated as non-zero.

b These entries are rms errors for values of $(R-I)_{\mathrm{C}}$ determined from $\beta, \alpha$, or $b-y$. An elementary form of the comparison algorithm has been used to determine these errors.

When the $[\mathrm{Fe} / \mathrm{H}]$ correction to temperatures derived from Balmer-line profiles is considered, it is found to differ from zero at $99.7 \%$ confidence (see Eq. (A.16)). For the data base used to derive the coefficient, moreover, the value of $|A|$ is only about 2 mmag dex ${ }^{-1}$. Apparently most of the $[\mathrm{Fe} / \mathrm{H}]$ correction comes from the Balmer-line temperatures specifically instead of contributing values of $(R-I)_{\mathrm{C}}$. This latter assessment is based on the Buser-Kurucz (1992) model-atmosphere calculations, so it should be re-evaluated as further calculations of that kind become available. 
Table A.4. Further color-index relations ${ }^{\mathrm{a}}$.

\begin{tabular}{|c|c|c|}
\hline $\begin{array}{l}\text { Equation } \\
\text { number }\end{array}$ & $\begin{array}{l}\text { Limits }^{\mathrm{b}} \\
\sigma_{\mathrm{c}}^{\mathrm{c}}\end{array}$ & Relation \\
\hline A. $11^{d}$ & $\begin{array}{l}0.17,0.40 \\
\quad 1.5\end{array}$ & $\begin{aligned}(R-I)_{\mathrm{C}} & =0.107+1.040(R-I)_{\mathrm{E}} \\
& \pm 0.001 \quad 0.008\end{aligned}$ \\
\hline A. $12^{\mathrm{e}}$ & $\begin{array}{l}0.10,0.50 \\
\quad 1.9\end{array}$ & 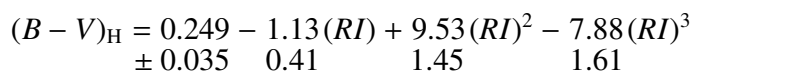 \\
\hline A. $13^{\mathrm{f}}$ & $\begin{array}{l}0.00,0.66 \\
\quad 5.4\end{array}$ & 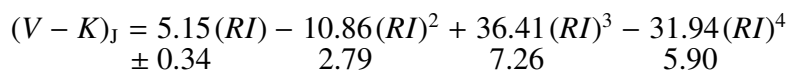 \\
\hline A. $14^{\mathrm{g}}$ & $\begin{array}{l}0.20,0.76 \\
1.2\end{array}$ & 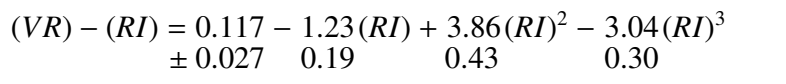 \\
\hline A. $15^{\mathrm{h}}$ & $0.30,0.60$ & $A=-0.083+0.342(R I)-0.296(R I)^{2}$ \\
\hline A. $16^{\mathrm{j}}$ & $\begin{array}{l}0.81,0.90 \\
0.0017\end{array}$ & $\begin{aligned} \theta=\theta_{\mathrm{BL}} & -0.025[\mathrm{Fe} / \mathrm{H}] \\
& \pm 0.008\end{aligned}$ \\
\hline A. $17^{\mathrm{k}}$ & $\begin{array}{l}0.28,0.85 \\
\quad 2.1\end{array}$ & $\begin{aligned}(R-I)_{\mathrm{C}} & =0.007+0.948\left(T_{1}-T_{2}\right) \\
& \pm 0.002 \quad 0.005\end{aligned}$ \\
\hline
\end{tabular}

${ }^{\text {a }}$ Units are magnitudes for all variables unless otherwise stated. For empirical (but not theoretical) relations, an rms error is given below each numerical coefficient.

b For each relation, the upper entries in this column are limits for $(R-I)_{\mathrm{C}}$ or $\theta$.

c For each relation, the lower entry in this column is the rms error of the centroid in mmag.

$\mathrm{d}$ This equation (from Taylor \& Joner 1988) applies only to post-1982 values of $(R-I)_{\mathrm{E}}$ for dwarfs which are rounded to the nearest $1 \mathrm{mmag}$.

e $(B-V)_{\mathrm{H}}$ is for the Hyades. Data sources for $(B-V)_{\mathrm{H}}$ are Johnson et al. (1962) and Mendoza $(1967)$. $(R I) \equiv(R-I)_{\mathrm{C}}$. Original source: Taylor (1994a, Sect. 2.2).

${ }^{\mathrm{f}}(V-K)_{\mathrm{J}}$ is Johnson $V-K .(R I) \equiv(R-I)_{\mathrm{C}}$. Data sources for $(V-K)_{\mathrm{J}}$ are Carney (1982) and Koornneef (1983). This relation is from Taylor (1992), and is for stars with $[\mathrm{Fe} / \mathrm{H}] \sim 0$.

g $(V R)-(R I) \equiv(V-R)_{\mathrm{C}}-(R-I)_{\mathrm{C}} \cdot(V-R)_{\mathrm{C}}$ is on the southern-hemisphere system, not the "Landolt subsystem" (Taylor \& Joner 1996). Original data source: Cousins (1978).

h $A=\partial(R-I)_{\mathrm{C}} / \partial[\mathrm{Fe} / \mathrm{H}]$, and is in $\operatorname{mag} \mathrm{dex}^{-1} .(R I) \equiv(R-I)_{\mathrm{C}}$. The rms residual of $A$ around the stated relation is 0.0029 . For $0.25 \leq(R I) \leq 0.3$, $A$ is assumed to be $0.035-0.14(R I)$. The relation is derived from calculations by Buser \& Kurucz (1992).

$\mathrm{j}[\mathrm{Fe} / \mathrm{H}]$ is in dex. $\theta \equiv 5040 / T(\mathrm{eff})$, and is unitless. $\theta_{\mathrm{BL}}$ is the raw temperature derived from Balmer-line profiles. Balmer-line data are from groups 2, 4, and 7 listed by Taylor (1992, Table 1). In practice, this relation is assumed to hold for $0.81 \leq \theta_{\mathrm{BL}} \leq 1.02$.

k This relation applies to the photometry of Gonzalez \& Piché (1992). For values of $(R-I)_{\mathrm{C}}$ derived from that source, $\sigma=20.7 n^{-0.5} \mathrm{mmag}$, with $n$ being the number of measurements.

\section{Appendix B: The solar color and temperature}

To establish a zero point for the adopted calibration, a solar value of the effective temperature $T_{\mathrm{e}}$ and a solar color index are required. For $T_{\mathrm{e}}$, the contributing quantities are as follows:

$F_{\text {bol }}(\min )=1365.6 \pm 0.16 \mathrm{~W} \mathrm{~m}^{-2}$,

$F_{\text {bol }}(\max )=1366.6 \pm 0.08 \mathrm{~W} \mathrm{~m}^{-2}$,

and

$\phi=1919.260$ arcsec.

These data are from White et al. (2002) and Di Benedetto (1998, Table 3). Equations (B.1) and (B.2) are from satellite measurements, and are assumed to be superior to results from ground-based flux measurements. The first of those equations applies at solar minimum, while the second applies at solar maximum.

Values of $T_{\mathrm{e}}$ are derived by substituting Eqs. (B.1) and (B.2) into the following familiar relation:

$T_{\mathrm{e}}^{4}=4 F_{\mathrm{bol}} / \phi^{2} \sigma_{\mathrm{SB}}$.
Here, $\sigma_{\mathrm{SB}}$ is the Stefan-Boltzmann constant. The result is

$T_{\mathrm{e}}=5776.4 \pm 0.3 \mathrm{~K}$,

with the quoted rms error including contributions from stated measurement error and from flux variations during the solar cycle. The corresponding value of $\theta_{\odot}$ is $0.87247 \pm 0.00005^{7}$.

The adopted color index for the Sun is a value of Cousins $R-I$ from Taylor (1997). That datum has been derived by using results from a number of stars and analysis methods. Taylor (1997, Sects. 3 through 6) has explained why it is preferable to use a low-blanketing color index like $(R-I)_{\mathrm{C}}$ in this context instead of $B-V$. A result from an analysis of broad scope is used because only such an analysis yields an adequate assessment of scatter among data from diverse techniques. As a result, only this kind of analysis yields a secure rms error (see Sect. 7 of Taylor 1997). By contrast, no rms error at all is available for a spectrum-synthesis result given by

7 To calculate this value of $\theta$, the numerical coefficient in the definition of $\theta$ is adopted with an increased number of significant figures: 5039.748, not 5040 . 
Bessell \& Brett (1988). Moreover, published solar color indices derived from solar analogs are based on measurements of no more than six stars (Bessell \& Norris 1984; Campins et al. 1985).

The value of the solar color index obtained by Taylor (1997) is

$(R-I)_{\mathrm{C}}=0.335 \pm 0.002 \mathrm{mag}$,

corresponding to $(V-K)_{\mathrm{J}}=1.474 \pm 0.012 \mathrm{mag}$. Adopting Eqs. (B.5) and (B.6), one finds that if their errors are added in quadrature, the net rms error in $\theta_{\odot}$ is 0.0022 . This error corresponds to $15 \mathrm{~K}$, and though it is included in the error budget used to calculate the catalog rms errors, it is in fact negligible.

\section{References}

Alonso, A., Arribas, S., \& Martinez Roger, C. 1996, A\&A, 313, 873

Bessell, M. 1983, PASP, 95, 480

Bessell, M., \& Norris, J. 1984, ApJ, 285, 622

Bessell, M., \& Brett, J. M. 1988, PASP, 100, 1134

Buser, R., \& Kurucz, R. L. 1992, A\&A, 264, 557

Campins, H., Rieke, G. H., \& Lebofsky, M. 1985, AJ, 90, 896

Carney, B. W. 1982, AJ, 87, 1527

Cayrel de Strobel, G., \& Cayrel, R. 1989, A\&A, 218, L9

Cousins, A. W. J. 1978, MNSSA, 37, 62

Cousins, A. W. J. 1980a, South Africa Astron. Obs. Circ., 1, 166

Cousins, A. W. J. 1980b, South Africa Astron. Obs. Circ., 1, 234

Crawford, D. L. 1975, AJ, 80, 955

Di Benedetto, G. P. 1998, A\&A, 339, 858

England, M. N. 1980, MNRAS, 191, 23

Fernley, J. A. 1989, MNRAS, 239, 905

Fuhrmann, K., Axer, M., \& Gehren, T. 1994, A\&A, 285, 585 (F94)

Fuhrmann, K. 1998, A\&A, 338, 161 (F98)

Fulbright, J. P. 2000, AJ, 120, 1841

Gonzalez, G. 1998, A\&A, 334, 221

Gonzalez, G., \& Laws, C. 2000, AJ, 119, 390

Gonzalez, G., \& Piché, F. 1992, AJ, 103, 2048

Gonzalez, G., \& Vanture, A. D. 1998, A\&A, 339, L29
Gonzalez, G., Wallerstein, G., \& Saar, S. H. 1999, ApJ, 511, L111

Gonzalez, G., Laws, C., Tyagi, S., \& Reddy, B. E. 2001, AJ, 121, 432

Gray, D. F. 1994, PASP, 106, 1248

Haywood, M. 2001, MNRAS, 325, 1365

Johnson, H. L., Mitchell, R. I., \& Iriarte, B. 1962, ApJ, 136, 75

Johnson, H. L., Mitchell, R. I., Iriarte, B., \& Wiśniewski, W. Z. 1966, Comm. Lunar and Planetary Lab., 4, 99

Kobi, D., \& North, P. 1990, A\&AS, 85, 999

Koornneef, J. 1983, A\&AS, 51, 489

Madansky, A. 1959, J. American Stat. Assoc., 54, 173

Mendoza, E. E. 1967, Bol. Tonantzintla Tacubaya, 4, 149

Mermilliod, J.-C., Mermilliod, M., \& Hauck, B. 1997, A\&AS, 124, 349

Raff, M. I. 1976, Ph.D. Thesis, University of California, Berkeley

Santos, M. C., Israelian, G., \& Mayor, M. 2001, A\&A, 373, 1019

Sekiguchi, M., \& Fukugita, M. 2000, AJ, 120, 1072

Spinrad, H., \& Taylor, B. J. 1969, ApJ, 157, 1279

Taylor, B. J. 1970, ApJS, 22, 177

Taylor, B. J. 1978, ApJS, 36, 173

Taylor, B. J. 1980, AJ, 85, 242

Taylor, B. J. 1986, ApJS, 60, 577

Taylor, B. J. 1991, ApJS, 76, 715

Taylor, B. J. 1992, PASP, 104, 500

Taylor, B. J. 1994a, PASP, 106, 444

Taylor, B. J. 1994b, PASP, 106, 452 (Paper I)

Taylor, B. J. 1994c, PASP, 106, 704

Taylor, B. J. 1995, PASP, 107, 734 (Paper II)

Taylor, B. J. 1996, ApJS, 102, 105

Taylor, B. J. 1997, in IAU Symp. 189, Fundamental Stellar Properties: The Interaction Between Observation and Theory, ed. T. R. Bedding, A. J. Booth, \& J. Davis (Dordrecht, Kluwer), 83

Taylor, B. J. 1999, A\&AS, 135, 75

Taylor, B. J. 2003, 398, 731

Taylor, B. J., \& Joner, M. D. 1988, AJ, 96, 211

Taylor, B. J., \& Joner, M. D. 1996, AJ, 111, 1338

Thoren, P., \& Feltzing, S. 2000, A\&A, 363, 692

White, O. R., de Toma, G., Chapman, G. A., et al. 2002, BAAS meeting 200, abstract 57.07 